\title{
On the Mechanics of Natural Compliance in Frictional Contacts and its Effect on Grasp Stiffness and Stability
}

\author{
Amir Shapiro \\ Dept. of ME, Technion
}

\author{
Elon Rimon \\ Dept. of ME, Technion
}

\author{
Joel W. Burdick \\ Dept. of ME, Caltech
}

\begin{abstract}
The mechanics of friction and compliance in multicontact arrangements is key to understanding and predicting grasp stability and dynamic response to external loads. This paper introduces a comprehensive model for the nonlinear forcedisplacement relationship at a frictional contact. The model is given in an analytic lumped parameter form suitable for on-line grasping applications, and is entirely determined by material and geometric properties of the contacting bodies. The forcedisplacement law predicts a nonlinear tangential stiffening as the normal load increases. As a result, the composite stiffness matrix of a frictional grasp is asymmetric, indicating that such grasps are not governed by any potential energy. The consequences for grasp stability are investigated. We formulate a rule for preloading frictional grasps which guarantees stable response at the individual contacts. Then we obtain a criterion for selecting contact points which guarantees overall grasp stability. The synthesis rule and its effect on grasp stability is illustrated with a simple 2D example.
\end{abstract}

\section{INTRODUCTION}

Friction effects play a key role in virtually all light to moderate duty grasping and manipulation applications. For instance, friction allows stable grasping with a much smaller number of contacts than would otherwise be required-two contacts rather than four in 2D, and two or three contacts rather than seven in 3D. Since friction effects act in tandem with natural compliance effects, the two phenomena must be integrated into a single comprehensive contact model. This paper has two main objectives. The first is to properly integrate friction and nonlinear compliance contact phenomena into a single law which would be theoretically justified by results from solid mechanics. The law must appear in an analytic lumped parameter form suitable for on-line grasping applications, with all its parameters being material coefficients and geometric parameters of the contacting bodies. The second objective is to analyze the stiffness and stability of multi contact grasps based on the friction-compliance model. This analysis ought to lead to synthesis rules indicating which contact points and what preloading profiles guarantee stable grasps.

The modeling of the nonlinear normal compliance is well understood in the robotics literature [1], [2], [3]. In contrast, there has been no systematic effort to incorporate results from the solid mechanics literature into a concise law that predicts the tangential force due to friction and natural compliance effects. Rather, roboticists have resorted to postulating ad-hoc linear springs that act tangentially in tandem with the rigidbody Coulomb friction law (e.g. [4], [5], [6]). However, the linear spring approach is not supported by the solid mechanics literature. In particular, without knowledge of an underlying nonlinear contact model there is no way to select the spring coefficients. Moreover, it is intuitively clear (and rigorously justified below) that the tangential stiffness increases as the bodies are pressed harder together. Yet none of the linearspring models proposed in the literature accounts for this phenomenon, which can potentially lead to grasp instability.

In the solid mechanics literature, the only focused effort on formulating analytical friction-compliance models is by Mindlin and Deresiewicz [7], [8][1949-1953]. They investigated the case where a contact is initially loaded along the normal direction in accordance with the Hertz normal compliance model. Then they analyze the tangential traction field generated by applying pure tangential loads while the normal penetration remains constant. Their investigation revealed highly nonlinear and complex phenomena such as micro-slip and hysteresis [9]. For almost half a century there has been no substantial progress in this area. Motivated by granular material packing applications, Walton [10] derived in 1987 an analytical friction-compliance model which is more relevant for grasping applications. This model was later refined by Elata [11]. Walton's law assumes that a contact is loaded along some relative linear motion between the contacting bodies. Under this assumption (and using a different analysis approach than Mindlin's), he derives a closed form formula for the tangential compliance force at a frictional contact. Our ensuing results are based on Walton's tangential compliance model.

The structure and contributions of the paper are as follows. In the next section we review Walton's contact model. Our description is limited to 2D bodies with spherical tips, but the model extends to 3D bodies. In Section III we compute the stiffness matrices associated with the individual contacts. These matrices, called the contact stiffness matrices, determine the composite stiffness matrix of the entire grasp. A fundamental new result is that the contact stiffiness matrices as well as the composite grasp stiffness matrix are non-symmetric. This result indicates that frictional grasps are not governed by any potential energy. Hence grasp stability must be determined by the full nonlinear dynamics of the contact arrangement, rather than being a simple local-minimum test. Section III also contains a characterization of the linear loading profiles that guarantee stability of the individual contact stiffness matrices. In Section IV we conduct a stability analysis of the full 


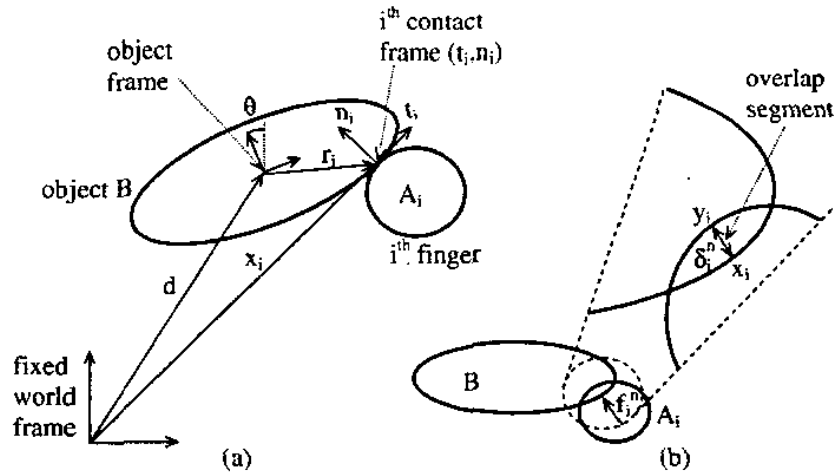

Fig. 1. (a) $\mathcal{B}$ 's c-space $\left(d_{x}, d_{y}, \theta\right)$. (b) The overlap segment between $\mathcal{A}_{i}$ and $\mathcal{B}$.

dynamics, and derive a concise grasp stability criterion that can be interpreted as a rule for selecting contact points that guarantees grasp stability. The concluding section discusses extension to $3 \mathrm{D}$ and on-going experimental validation of the model predictions.

\section{Friction-COMPLIANCE MODELS}

We describe generic nonlinear models for the normal and tangential compliance at a frictional contact. Consider a grasp or fixture arrangement where a $2 \mathrm{D}$ object $\mathcal{B}$ is held by stationary $2 \mathrm{D}$ bodies $\mathcal{A}_{1}, \ldots, \mathcal{A}_{k}$ which represent fingertips or fixels. The usual assumption made in the solid mechanics literature is that the contacting bodies are quasi-rigid, meaning that their deformations due to compliance effects are localized to the vicinity of the contacts [9]. This assumption is generally valid for all bodies which are not made of exceptionally soft material and do not contain slender substructures [3]. The quasi-rigidity assumption allows us to describe the overall motion of $\mathcal{B}$ relative to the stationary bodies $\mathcal{A}_{1}, \ldots, \mathcal{A}_{k}$ using rigid body kinematics. Since the grasping bodies are stationary, we focus on $\mathcal{B}$ 's configuration space (c-space). C-space is parametrized by $q=(d, \theta) \in \mathbb{R}^{2} \times \mathbb{R}$, where $d$ is $\mathcal{B}$ 's position and $\theta$ is a parametrization of $\mathcal{B}$ 's orientation. Velocity vectors take the form $\dot{q}=(v, \omega)$, where $v \in \mathbb{R}^{3}$ and $\omega \in \mathbb{R}^{3}$ are the linear and angular velocities of $\mathcal{B}$.

\section{A. Normal Compliance Models}

We review a generic normal compliance modeling approach that ignores the details of compliant surface deformations and models the resultant contact force as a function of $\mathcal{B}$ 's configuration [2]. Consider a single contact between $\mathcal{B}$ and $\mathcal{A}_{i}$. In the absence of deformation, the two bodies contact at a single point. When pushed together, the two contacting surfaces deform. One can conceptually think of the two rigid bodies inter-penetrating, or overlapping their undeformed shapes, as illustrated in Figure 1(b). Let $\mathcal{B}$ be at a configuration $q$. Then the overlap ${ }^{1}$ between $\mathcal{B}$ and $\mathcal{A}_{i}$, denoted $\delta_{i}^{n}(q)$, is the minimum amount of translation of $\mathcal{B}$ that would separate it

\footnotetext{
${ }^{1}$ The notion of overlap used here is consistent with the concept of "relative approach" in contact mechanics [9].
}
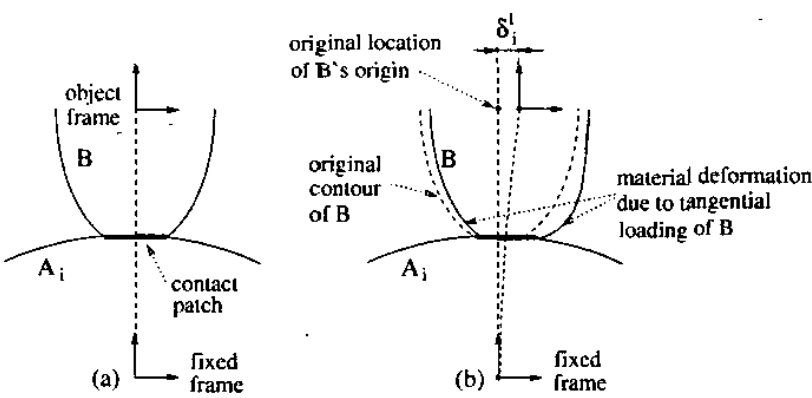

Fig. 2. (a) An initial contact area generated by normal loading of $\mathcal{B}$ against $\mathcal{A}_{i}$. (b) Tangential loading of $\mathcal{B}$ causes tangential displacement of $\mathcal{B}$ without any macro-slip.

from $\mathcal{A}_{i}$. The overlap segment is the unique segment whose endpoints lie on the boundary of $\mathcal{B}$ and $\mathcal{A}_{i}$, such that the length of the segment is $\delta_{i}^{n}$ and its orientation gives the direction of separating translation. For sufficiently small $\delta_{i}^{n}$, the overlap segment is collinear with the normals to the boundaries of $\mathcal{B}$ and $\mathcal{A}_{i}$. In this lumped parameter form of modeling, the net normal force induced by the local deformation is assumed to act at $\mathcal{B}$ 's endpoint of the overlap segment, $x_{i}$, in the direction of the overlap segment. The magnitude of the net normal force, $f_{i}^{n}$, is assumed to depend on $\delta_{i}^{n}$ in terms of a function $g_{i}\left(\delta_{i}^{n}\right)$. This function is required to be differentiable, zero when $\delta_{i}^{n}$ is zero, and monotonically increasing when $\delta_{i}^{n}$ is positive. The normal compliant force is:

$$
\begin{aligned}
f_{i}^{n}(q)= & g_{i}\left(\delta_{i}^{n}(q)\right)+\varphi_{i}^{n}\left(\delta_{i}^{t}, \delta_{i}^{n}, \dot{\delta}_{i}^{n}\right) \\
& \text { s.t. } g_{i}^{\prime}\left(\delta_{i}^{n}\right)>0 \text { when } \delta_{i}^{n}>0 .
\end{aligned}
$$

The function $\varphi_{i}^{n}$ represents damping due to viscoelastic effects. It is differentiable, $\varphi_{i}^{n}\left(\delta_{i}^{t}, \delta_{i}^{n}, 0\right)=0$, and being damping function satisfies the condition $\varphi_{i}^{n}\left(\delta_{i}^{t}, \delta_{i}^{n}, \dot{\delta}_{i}^{n}\right) \cdot \dot{\delta}_{i}^{n}<0$. It is important to note that a wide variety of contact models can be represented in this framework. The simplest contact model assumes that $g_{i}$ is a linear function of the overlap: $g_{i}\left(\delta_{i}^{n}\right)=\kappa_{i} \delta_{i}^{n}$, where the coefficient $\kappa_{i}$ represents the combined stiffness of $\mathcal{B}$ and $\mathcal{A}_{i}$ at the contact [1]. The nonlinear Hertz model [12] which has been verified theoretically and experimentally, establishes that quasi-rigid bodies with spherical tips of radii $R_{1}$ and $R_{2}$ satisfy the law:

$$
f_{i}^{n}=g_{i}\left(\delta_{i}^{n}\right)=\frac{8 G \sqrt{R}}{3(1-\nu)}\left(\delta_{i}^{n}\right)^{3 / 2},
$$

where $R=\frac{R_{1} R_{2}}{R_{1}+R_{2}}$, and $G$ and $\nu$ are material shear modulus and Poisson's ratio [9]. The overlap representation is useful even for modeling bodies which are not necessarily quasirigid, such as soft fingertips [13].

\section{B. Tangential Compliance Models}

The process underlying tangential compliance at a frictional contact is as follows. When two quasi-rigid bodies are preloaded along the normal direction, they locally deform and establish a contact area centered at the original contact point 
(Figure 2(a)). The deformed bodies generate a normal forcefield which is continuously distributed along the contact area. (The integral of this force-field over the contact area gives the net normal force described above.) When the two bodies are next loaded along a tangential direction, they locally deform in a way that generates a tangential force-field which is again continuously distributed along the contact area (Figure 2(b)). The usual assumption made in the solid mechanics literature is that the normal and tangential force-fields interact at the individual points of the contact area according to Coulomb's law [8]. Under this assumption, elasticity theory as well as experimentals indicate that the tangent force-field consists of two regimes. At points in an outer ring of the contact area the tangent forces exceed the friction cone constraint, causing micro-slip at these points. At points along the complementary inner disc of the contact area the tangent forces lie within the friction cone, and at these points no micro-slip takes place. As the magnitude of the tangential loading increases the area of the stationary inner disc shrinks. Finally, when the net tangential loading reaches $\mu$ times the net normal loading ( $\mu$ being the coefficient of friction), the inner disc shrinks to a point and the two bodies experience macro-slip at the contact.

Based on this insight, we formulate a generic tangential compliance law, assuming that the contacting bodies deform but do not slip. This law covers most of the tangential compliance models that have been proposed in the literature [11], [8], [10], and is therefore quite general. First we need to introduce notation. Recall that $x_{i}$ is $\mathcal{B}$ 's endpoint of the overlap segment. Let $r_{i}$ denote the same point expressed in $\mathcal{B}$ 's body frame (Figure 1(a)). Then $x_{i}$ is related to $r_{i}$ by the rigid body transformation: $x_{i}=X\left(r_{i}, q\right)=R(\theta) r_{i}+d$, where $R(\theta)$ is the orientation matrix of $\mathcal{B}$. Let $X_{r_{i}}(q)$ denote the rigid body transformation with $r_{i}$ held fixed. When $\mathcal{B}$ moves along a c-space trajectory $q(t)$, the velocity of $X_{r_{i}}$ is given by $\frac{d}{d t} X_{r_{i}}(q(t))=G_{i}^{T} \dot{q}(t)$, where $G_{i}^{T}=D X_{r_{i}}$ is the $2 \times 3$ Jacobian matrix of $X_{r_{i}}$. Now let $\delta_{i}^{t}(q(t))$ denote the tangential displacement of $\mathcal{B}$ relative to the $i^{\text {th }}$ contact due to motion of $\mathcal{B}$ (Figure 2(b)). Then the derivative of $\delta_{i}^{t}$ along $q(t)$ is given by projection of the velocity of $X_{r_{i}}$ along the unit vector $t_{i}$ which is orthogonal to the current overlap segment:

$$
\frac{d}{d t} \delta_{i}^{t}(q(t))=-t_{i} \cdot \dot{X}_{r_{i}}=-t_{i} \cdot G_{i}^{T} \dot{q}(t) .
$$

Note that in contrast with $\delta_{i}^{n}(q)$, the tangential displacement is not a direct function of $q$, but requires integration of (2) over the entire loading trajectory [9][p. 221].

The magnitude of the net tangential force, $f_{i}^{t}$, obeys a generic law of the form:

$$
\begin{aligned}
f_{i}^{t}= & h_{i}\left(\delta_{i}^{t}, \delta_{i}^{n}\right)+\varphi_{i}^{t}\left(\delta_{i}^{t}, \delta_{i}^{n}, \dot{\delta}_{i}^{t}\right) \\
& \quad \text { as long as } f_{i}^{n}>0 \text { and }\left|f_{i}^{t}\right| \leq \mu f_{i}^{n},
\end{aligned}
$$

where $\mu$ is the coefficient of friction. The function $h_{i}$ represents the elastic part of the tangential force. It is differentiable, $h_{i}\left(0, \delta_{i}^{n}\right)=0$, and for any fixed positive $\delta_{i}^{n}$ is monotonically increasing in $\delta_{i}^{t}$. Note that $h_{i}$ depends both on the tangential and normal displacements $\delta_{i}^{t}$ and $\delta_{i}^{n}$. The function $\varphi_{i}^{t}$ represents damping due to micro-slip. It is differentiable, $\varphi_{i}^{t}\left(\delta_{i}^{t}, \delta_{i}^{n}, 0\right)=0$, and satisfies the condition $\varphi_{i}^{t}\left(\delta_{i}^{t}, \delta_{i}^{n}, \dot{\delta}_{i}^{t}\right) \cdot \dot{\delta}_{i}^{t}<$ 0 .

Walton's tangential compliance model. Walton assumes that a contact is loaded along a linear loading profile satisfying $\delta_{i}^{t}=c_{i} \delta_{i}^{n}$ such that $c_{i}$ is constant throughout the loading process. Under this assumption, he derives the following formula for the elastic part of the tangential compliance force [10],

$$
\begin{aligned}
f_{i}^{t}=h_{i}\left(\delta_{i}^{t}, \delta_{i}^{n}\right)= & \frac{16 G \sqrt{R}}{3(2-\nu)} \sqrt{\delta_{i}^{n}} \delta_{i}^{t} \\
& \text { such that }\left|c_{i}\right| \leq \mu \frac{(2-\nu)}{2(1-\nu)} .
\end{aligned}
$$

The condition $\left|c_{i}\right| \leq \mu \frac{(2-\nu)}{2(1-\nu)}$ results from substituting formulas (1) and (3) for $f_{i}^{n}$ and $f_{i}^{t}$ in the friction cone constraint $\left|f_{i}^{t}\right| \leq \mu f_{i}^{n}$. Walton's formula is extremely relevant for grasping applications, since one can readily implement a linear preloading of the grasp's contacts. As noted in the introduction, Walton's formula indicates a nonlinear tangential stiffening at a contact for larger normal penetrations.

\section{The Contact STIFfness Matrix}

Let $q_{0}$ denote the configuration of $\mathcal{B}$ at the preloaded grasp. The contact stiffness matrix, $K_{i}$, is the $2 \times 2$ matrix representing the linearized force-displacement relationship at the contact:

$$
\left(\begin{array}{c}
\Delta f_{i}^{t} \\
\Delta f_{i}^{\mathrm{n}}
\end{array}\right)=K_{i}\left(q_{0}\right)\left(\begin{array}{c}
\Delta \delta_{i}^{t} \\
\Delta \delta_{i}^{\mathrm{n}}
\end{array}\right) .
$$

We wish to derive a formula for $K_{i}$ based on the HertzWalton model. However, Walton's model is valid only for linear loading profiles. Hence the linearized Walton law would give the tangential force corresponding to a linear loading profile which leads directly to $\left(\delta_{i}^{t}\left(q_{0}\right), \delta_{i}^{n}\left(q_{0}\right)\right)+\left(\Delta \delta_{i}^{t}, \Delta \delta_{i}^{n}\right)$. On the other hand, $K_{i}$ in (4) gives the contact force obtained by first loading along a linear profile towards $\left(\delta_{i}^{t}\left(q_{0}\right), \delta_{i}^{n}\left(q_{0}\right)\right)$, then loading along a small change $\left(\Delta \delta_{i}^{t}, \Delta \delta_{i}^{n}\right)$. In order to obtain a formula for $K_{i}$, we introduce a practical assumption that closely matching loading profiles generate closely matching tangential traction fields. While a formal justification of this assumption is under investigation, it is clearly a reasonable assumption. Under this continuity-with-respect-toloading-profile assumption, the formula for $K_{i}$ is precisely the linearized Hertz-Walton laws. The following proposition gives the formula.

Proposition III.1. Let two quasi-rigid bodies with spherical tips of radii $R_{1}$ and $R_{2}$ be preloaded along a linear path $\delta_{i}^{t}(q)=c_{i} \delta_{i}^{n}(q)$ such that $\left|c_{i}\right| \leq \mu \frac{(2-\nu)}{2(1-\nu)}$. Then the stiffness matrix of the loaded contact is:

$$
K_{i}=4 G \sqrt{R \delta_{i}^{n}\left(q_{0}\right)}\left[\begin{array}{cc}
\frac{4}{3(2-\nu)} & \frac{2 c_{i}}{3(2-\nu)} \\
0 & \frac{1}{1-\nu}
\end{array}\right],
$$

where $\delta_{i}^{n}\left(q_{0}\right)$ is the normal penetration, $R=\frac{R_{1} R_{2}}{R_{1}+R_{2}}$, and $G$ and $\nu$ are material shear modulus and Poisson's ratio. 
The formula for $K_{i}$ is obtained by first taking the derivative of $\left(f_{i}^{n}, f_{i}^{t}\right)$ given in (1) and (3) with respect to $\left(\delta_{i}^{n}, \delta_{i}^{t}\right)$. Then substituting $q \doteq q_{0}$ and the loading path relation $\delta_{i}^{t}\left(q_{0}\right)=$ $c_{i} \delta_{i}^{n}\left(q_{0}\right)$. Note that the resulting contact stiffness matrix is asymmetric. We shall see below that the composite stiffness matrix of the entire grasp is consequently asymmetric.

For purposes of grasp stability analysis, we need to establish when the symmetric part of $K_{i}$ is positive definite. (This condition was characterized as a "stable" $K_{i}$ in the introduction.) Let $\left(K_{i}\right)_{s}=\frac{1}{2}\left(K_{i}+K_{i}^{T}\right)$ denote the symmetric part of $K_{i}$, and let $\left(K_{i}\right)_{a s}=\frac{1}{2}\left(K_{i}-K_{i}^{T}\right)$ denote the skew-symmetric part of $K_{i}$, where $K_{i}=\left(K_{i}\right)_{s}+\left(K_{i}\right)_{a s}$. A surprising result is that the positive definiteness of $\left(K_{i}\right)_{s}$ depends solely on the slope of the linear loading profile.

Proposition III.2. If a contact is loaded along a linear loading profile whose slope $c_{i}$ satisfies

$$
\left|c_{i}\right|<2 \sqrt{\frac{3(2-\nu)}{(1-\nu)}}
$$

the symmetrized contact stiffiness matrix $\left(K_{i}\right)_{s}$ is positive definite.

Proof: Let $\lambda_{1}, \lambda_{2}$ denote the eigenvalues of $\left(K_{i}\right)_{s}$. Then $\left(K_{i}\right)_{s}$ is positive definite iff $\lambda_{1}, \lambda_{2}>0$. First consider the trace of $\left(K_{i}\right)_{s}$. The trace is positive when $\nu<1$. But for almost all practical materials Poisson's ratio $\nu$ is bounded from above by 0.5 [9]. Hence $\operatorname{tr}\left(K_{i}\right)_{s}=\lambda_{1}+\lambda_{2}>0$. Next we compute the determinant $\operatorname{det}\left(K_{i}\right)_{s}=\lambda_{1} \lambda_{2}$. Since $\lambda_{1}+\lambda_{2}>0$, the positive definiteness of $\left(K_{i}\right)_{s}$ would follow from the condition $\lambda_{1} \lambda_{2}>0$. Ignoring the positive coefficient $4 G \sqrt{R \delta_{i}^{n}\left(q_{0}\right)}$, the determinant of $\left(K_{i}\right)_{s}$ is:

$$
\operatorname{det}\left(K_{i}\right)_{s}=\frac{4}{3(1-\nu)(2-\nu)}-\frac{c_{i}^{2}}{9(2-\nu)^{2}} .
$$

The inequality $\operatorname{det}\left(K_{i}\right)_{s}>0$ becomes $4 / 3(1-\nu)(2-\nu)>$ $c_{i}^{2} / 9(2-\nu)^{2}$. Taking the square root of both sides gives the result.

Example: For typical values of $\nu \leq 0.5$, the loading path slope must satisfy $\left|c_{i}\right| \leq 6$ for $\left(K_{i}\right)_{s}$ to be positive definite. The corresponding angle, denoted $\beta$ in Figure 3 , must satisfy $\beta \leq 80.5^{\circ}$. However, $c_{i}$ must also satisfy the friction cone constraint $\left|c_{i}\right| \leq \mu(2-\nu) / 2(1-\nu)$. For typical values of $\mu \leq 1$, the loading path slope must satisfy $\left|c_{i}\right| \leq 1.5$. The corresponding angle, denoted $\alpha$ in Figure 3, must satisfy $\alpha \leq$ $56.3^{\circ}$. Thus we see that the positive definiteness requirement is significantly less restrictive than the friction cone constraint. Since friction cone constraint must always be satisfied, we conclude that $\left(K_{i}\right)_{s}$ is typically positive definite.

\section{STABILITy ANALysis}

Our objective is to determine the stability of frictional grasps under the Hertz-Waiton model. We first derive the linearized dynamics of $\mathcal{B}$. The resulting system contains an asymmetric stiffens matrix. Hence we develop a general stability criterion for asymmetric linear systems, then applied the criterion to our grasping system.

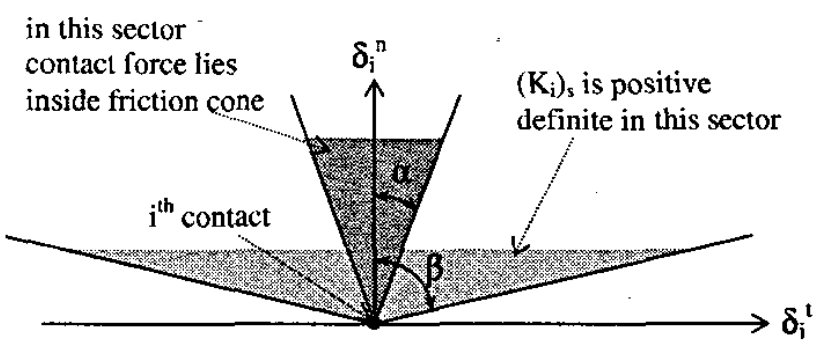

Fig. 3. Two sectors in $\left(\delta_{i}^{t}, \delta_{i}^{n}\right)$-plane associated with positive definiteness of $\left(K_{i}\right)_{s}$ and friction cone constraint. Typically $\alpha \leq 56.3^{\circ}, \beta \leq 80.5^{\circ}$.

\section{A. Linearized Grasp Dynamics}

We derive the linearized dynamics of a quasi-rigid object $\mathcal{B}$ held in equilibrium grasp by stationary quasi-rigid bodies $\mathcal{A}_{1}, \ldots, \mathcal{A}_{k}$ under two assumptions. First, we assume that the bodies have spherical tips at the contacts. Second, we assume that each contact is preloaded along a linear loading profile.

Let $F_{i}$ denote the $i^{\text {th }}$ finger force acting on $\mathcal{B}$, where $F_{i}$ is expressed in a fixed world frame. Since $\left(f_{i}^{t}(q, \dot{q}), f_{i}^{n}(q)\right)$ are the tangential and normal components of $F_{i}$, we write $F_{i}$ as $F_{i}(q, \dot{q})$. The formula for $F_{i}$ in terms of $\left(f_{i}^{t}, f_{i}^{n}\right)$ is $F_{i}=$ $R_{i}(q)\left(\begin{array}{c}f_{i}^{t} \\ f_{i}^{n}\end{array}\right)$, where $R_{i}(q)$ is the $2 \times 2$ matrix $R_{i}(q)=\left[t_{i} n_{i}\right]$. Next consider the wrench (i.e. force and torque) induced by $F_{i}$ on $\mathcal{B}$. It can be verified that this wrench is given by $G_{i}(q) F_{i}(q, \dot{q})$, where $G_{i}=D X_{r_{i}}^{T}$ was introduced above. Using this notation, the dynamics of $\mathcal{B}$ under the influence of $k$ contact forces, without any other external influences such as gravity, is:

$$
M(q) \ddot{q}+C(q, \dot{q})=\sum_{i=1}^{k} G_{i}(q) F_{i}(q, \dot{q}),
$$

where $M(q)$ and $C(q, \dot{q})$ are $\mathcal{B}$ 's $3 \times 3$ inertia matrix and vector of centrifugal and Coriolis forces.

Recall that $q_{0}$ denotes the equilibrium grasp configuration of $\mathcal{B}$. We wish to determine the linearized dynamics of $\mathcal{B}$ at the equilibrium state $(q, \dot{q})=\left(q_{0}, 0\right)$. Let $\left(p_{1}, p_{2}\right)=(q, \dot{q})$ denote the state variables. Then $(5)$ is given by

$$
\begin{aligned}
& \dot{p}_{1}=p_{2} \\
& \dot{p}_{2}=M^{-1}\left(p_{1}\right)\left(\sum_{i=1}^{k} G_{i}\left(p_{1}\right) F_{i}\left(p_{1}, p_{2}\right)-C\left(p_{1}, p_{2}\right)\right) .
\end{aligned}
$$

The following lemma gives the linearized dynamics of $\mathcal{B}$ at the equilibrium state.

Lemma IV.1. The linearized dynamics of $\mathcal{B}$ at $\left(p_{1}, p_{2}\right)=$ $\left(q_{0}, 0\right)$ is given by

$$
\left(\begin{array}{c}
\Delta p_{1} \\
\Delta p_{2}
\end{array}\right)=\left[\begin{array}{cc}
0 & I \\
-M^{\sim 1}\left(q_{0}\right) K_{p}\left(q_{0}\right) & -M^{-1}\left(q_{0}\right) K_{d}\left(q_{0}\right)
\end{array}\right]\left(\begin{array}{l}
\Delta p_{1} \\
\Delta p_{2}
\end{array}\right)
$$

where $K_{p}$ and $K_{d}$ are the grasp $3 \times 3$ stiffness and damping matrices. The stiffness matrix is given by

$$
K_{p}=\sum_{i=1}^{k} G_{i} R_{i} K_{i} R_{i}^{T} G_{i}^{T}-\left(D G_{i}^{T}+\left(D R_{i}\right) R_{i}^{T}\right) F_{i}
$$


where $K_{i}$ is the $i^{\text {th }}$ contact stiffness matrix. The damping matrix is given by

$$
K_{d}=-\sum_{i=1}^{k} G_{i} R_{i}\left[\begin{array}{cc}
\frac{\partial \varphi_{i}^{\ell}}{\partial \dot{\delta}_{i}^{t}} & 0 \\
0 & \frac{\partial \varphi_{i}^{n}}{\partial \dot{\delta}_{i}^{n}}
\end{array}\right] R_{i}^{T} G_{i}^{T},
$$

where $\varphi_{i}$ is the $i^{\text {th }}$ tangential damping function.

The detailed proof is relegated to Ref. [14]. In the proof, we first observe that the linearization of $M\left(p_{1}\right)$ and $C\left(p_{1}, p_{2}\right)$ vanishes at the equilibrium state. Then we focus on the matrices: $K_{p}=\left.\frac{\partial}{\partial p_{1}}\right|_{\left(p_{1}, p_{2}\right)=\left(q_{0}, 0\right)} \sum_{i=1}^{k} G_{i}\left(p_{1}\right) F_{i}\left(p_{1}, p_{2}\right)$, and $K_{d}=\left.\frac{\partial}{\partial p_{2}}\right|_{\left(p_{1}, p_{2}\right)=\left(g_{0}, 0\right)} \sum_{i=1}^{k} G_{i}\left(p_{1}\right) F_{i}\left(p_{1}, p_{2}\right)$, where $\Delta p_{1}=$ $\Delta q=q-q_{0}$, and $\Delta p_{2}=\dot{q}-0$. Note that the linearized grasp dynamics can also be written as

$$
M\left(q_{0}\right) \Delta \ddot{q}+K_{d}\left(q_{0}\right) \Delta \dot{q}+K_{p}\left(q_{0}\right) \Delta q=0,
$$

such that $M\left(q_{0}\right)$ and $K_{d}\left(q_{0}\right)$ are symmetric matrices, while $K_{p}\left(q_{0}\right)$ is asymmetric.

\section{B. Stability of $2^{\text {nd }}$-Order Asymmetric Linear Systems}

Consider the second order linear asymmetric system:

$$
\ddot{p}+K_{d} \dot{p}+K_{p} p=0
$$

where $K_{d} \in \mathbb{R}^{n \times n}$ is symmetric positive definite, $K_{p} \in$ $\mathbb{R}^{n \times n}$ is asymmetric, while its symmetric part $\left(K_{p}\right)_{s}$ is positive definite. The following theorem states that if the skewsymmetric part of $K_{p},\left(K_{i}\right)_{a s}$, is sufficiently small, the system (7) is globally asymptotically stable.

Theorem 1 (global asymptotic stability). Consider the system of (7). Let $\beta \in \mathbb{R}$ be the minimal eigenvalue of $K_{d}$. Let $\alpha \in \mathbb{R}$ be the minimal eigenvalue of $\left(K_{p}\right)_{s}$, and let $\gamma \in \mathbb{R}$ be the matrix norm ${ }^{2}$ of the skew-symmetric part of $K_{p}$. Then if

$$
|\gamma|<\sqrt{\alpha} \beta
$$

the system (7) is globally asymptotically stable.

Proof: The system (7) can be written as

$$
\frac{d}{d t}\left(\begin{array}{l}
p \\
\dot{p}
\end{array}\right)=\underbrace{\left[\begin{array}{cc}
0 & I \\
-K_{p} & -K_{d}
\end{array}\right]}_{A}\left(\begin{array}{l}
p \\
\dot{p}
\end{array}\right)
$$

For global asymptotic stability, it suffices to show that the real part of the eigenvalues of $A$ is negative. Let $\lambda \in \mathbb{C}$ be an eigenvalue of $A$ with corresponding eigenvector $v=$ $\left(v_{1}, v_{2}\right) \in \mathbb{C}^{2 n}(v \neq 0)$. Note that each $v_{i}$ is a complex vector in $\mathbb{C}^{n}$. Then

\footnotetext{
${ }^{2}$ The matrix norm is defined as $\|E\|=\max \{\|E u\|\}$ over all vectors $\|u\| \leq 1$.
}

$$
\begin{gathered}
{\left[\begin{array}{cc}
0 & I \\
-K_{p} & -K_{d}
\end{array}\right]\left(\begin{array}{c}
v_{1} \\
v_{2}
\end{array}\right)} \\
\quad=\left(\begin{array}{c}
v_{2} \\
-K_{p} v_{1}-K_{d} v_{2}
\end{array}\right)=\lambda\left(\begin{array}{l}
v_{1} \\
v_{2}
\end{array}\right) .
\end{gathered}
$$

Clearly, $\lambda=0$ cannot be an eigenvalue of $A$. Since $\lambda \neq 0$, it follows that $v_{1} \neq \overrightarrow{0}$ and $v_{2} \neq \overrightarrow{0}$. Hence we may assume without loss of generality that $v_{1}^{*} \cdot v_{1}=1$, where $*$ denotes complex conjugate transpose. Based on this choice, we can write $\lambda^{2}=v_{1}^{*} \lambda^{2} v_{1}=v_{1}^{*} \lambda v_{2}=v_{1}^{*}\left(-K_{p} v_{1}-K_{d} v_{2}\right)=$ $-v_{1}^{*} K_{p} v_{1}-\lambda v_{1}^{*} K_{d} v_{1}$, where we used the relations $\lambda v_{1}=v_{2}$ and $\lambda v_{2}=-K_{p} v_{1}-K_{d} v_{2}$. Since $K_{d}>0$, the scalar $\tilde{\beta}=$ $v_{1}^{*} K_{d} v_{1}$ is positive real. Similarly, the scalar $\tilde{\alpha}=v_{1}^{*}\left(K_{p}\right)_{s} v_{1}$ is also positive real. Since $\left(K_{p}\right)_{a s}$ is skew-symmetric, we can write $j \tilde{\gamma}=v_{1}^{*}\left(K_{p}\right)_{a s} v_{1}$, where $j=\sqrt{-1}$ and $\tilde{\gamma}$ is real. Substituting these scalars into the quadratic equation of $\lambda$ gives

$$
\lambda^{2}+\tilde{\beta} \lambda+\tilde{\alpha}+j \tilde{\gamma}=0 \text {. }
$$

Note that every eigenvalue of $A$ satisfies this equation. The solution of $(8)$ is:

$$
\lambda_{1,2}=\frac{1}{2}\left(-\tilde{\beta} \pm \sqrt{\tilde{\beta}^{2}-4(\tilde{\alpha}+j \tilde{\gamma})}\right) .
$$

Let us pause to recall how one computes the square root of a complex number. Consider a complex number $z=a+j b$ with a norm $|z|=\sqrt{a^{2}+b^{2}}$ and argument $\theta=\arctan (b / a)$. Then $\sqrt{z}= \pm\left(a^{2}+b^{2}\right)^{\frac{1}{4}} \angle \frac{\theta}{2}$, and in cartesian coordinates $\sqrt{z}=$ $\pm\left(a^{2}+b^{2}\right)^{\frac{1}{4}}\left(\cos \left(\frac{\theta}{2}\right)+j \sin \left(\frac{\theta}{2}\right)\right)$. Since $\cos (\theta)=\frac{a}{\sqrt{a^{2}+b^{2}}}$, we use the trigonometric identity $\cos \left(\frac{\theta}{2}\right)=\sqrt{\frac{1+\cos (\theta)}{2}}$ to obtain

$$
\operatorname{Re}\{\sqrt{z}\}= \pm \frac{\left(a^{2}+b^{2}\right)^{\frac{1}{4}}}{\sqrt{2}}\left(1+\frac{a}{\sqrt{a^{2}+b^{2}}}\right)^{\frac{1}{2}} .
$$

In our case $a=\tilde{\beta}^{2}-4 \tilde{\alpha}$ and $b=-4 \tilde{\gamma}$, and (9) implies that

$$
\begin{aligned}
& \operatorname{Re}\left\{\lambda_{1,2}\right\}=-\frac{\bar{\beta}}{2} \\
& \pm \frac{\left(\left(\tilde{\beta}^{2}-4 \bar{\alpha}\right)^{2}+16 \tilde{\gamma}^{2}\right)^{\frac{1}{4}}}{2 \sqrt{2}}\left(1+\frac{\left(\tilde{\beta}^{2}-4 \tilde{\alpha}\right)}{\sqrt{\left(\tilde{\beta}^{2}-4 \bar{\alpha}\right)^{2}+16 \bar{\gamma}^{2}}}\right)^{\frac{1}{2}}
\end{aligned}
$$

The requirement $\operatorname{Re}\left\{\lambda_{1,2}\right\}<0$ introduces an inequality in $\tilde{\alpha}, \tilde{\beta}$, and $\tilde{\gamma}$. Rearranging terms in this inequality gives the equivalent inequality,

$$
\left(4 \bar{\alpha}+\tilde{\beta}^{2}\right)^{2}>\left(\tilde{\beta}^{2}-4 \tilde{\alpha}\right)^{2}+16 \tilde{\gamma}^{2} .
$$

Cancelling similar terms yields the inequality

$$
|\tilde{\gamma}|<\sqrt{\tilde{\alpha}} \tilde{\beta}
$$

For stability we must ensure that the inequality (10) holds for every $\tilde{\alpha}, \tilde{\beta}$, and $\bar{\gamma}$. In other words, (10) must hold for every eigenvalue $\lambda$ and associated eigenvector $v$ of $A$. Therefore we bound $\tilde{\alpha}, \tilde{\beta}$, and $\tilde{\gamma}$ as follows. First, $0<\alpha=\lambda_{\min }\left(\left(K_{p}\right)_{s}\right) \leq$ $v_{1}^{*}\left(K_{p}\right)_{s} v_{1}=\tilde{\alpha}$. Second, $0<\beta=\lambda_{\min }\left(K_{d}\right) \leq v_{1}^{*} K_{d} v_{1}=$ 


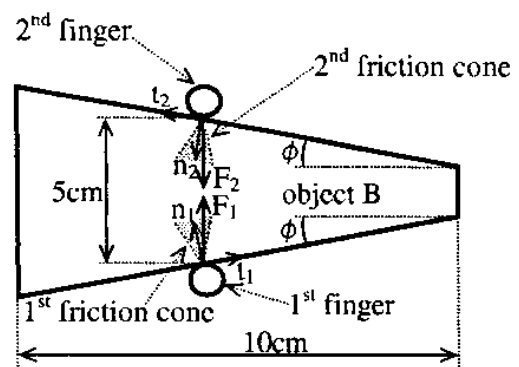

Fig. 4. An example of two-fingered grasp. where the fingers radii are $1[\mathrm{~cm}]$.

$\tilde{\beta}$. Third, $|\gamma|=\left\|\left(K_{p}\right)_{a s}\right\| \geq\left|v_{1}^{*}\left(K_{p}\right)_{a s} v_{1}\right|=|j \bar{\gamma}|=|\bar{y}|$ Using these bounds, $\gamma<\sqrt{\alpha} \beta$ implies that $|\tilde{\gamma}|<\sqrt{\tilde{\alpha}} \tilde{\beta}$ for every $\tilde{\alpha}, \tilde{\beta}$, and $\tilde{\gamma}$.

Application to grasp stability. The linearized grasp system contains an additional term, the inertia matrix $M\left(q_{0}\right)$ which multiplies $\ddot{p}$. The following corollary adapts the theorem to a global asymptotic stability criterion for the linearized grasp system.

\section{Corollary IV.2. Consider the linearized grasp system}

$$
M \ddot{p}+K_{d} \dot{p}+K_{p} p=0,
$$

where all parameters are as above, except for the matrix $M \in$ $\mathbb{R}^{n \times n}$ which is symmetric positive definite. Let $\beta>0$ be the minimal eigenvalue of $M^{-1 / 2} K_{d} M^{-1 / 2}$. Let $\alpha>0$ be the minimal eigenvalue of $M^{-1 / 2}\left(K_{p}\right)_{s} M^{-1 / 2}$, and let $\gamma \in \mathbb{R}$ be the matrix norm of $M^{-1 / 2}\left(K_{p}\right)_{a s} M^{-1 / 2}$. Then if

$$
|\gamma|<\sqrt{\alpha} \beta
$$

the system (II) is globally asymptotically stable.

The proof of the corollary appears in Ref. [14]. We conclude this section with an example of a two-finger grasp frictional of a polygonal object.

\section{Two-Fingered Grasp Example}

Consider the case of trapezoidal object of $1[\mathrm{~cm}]$ thickness, grasped by two fingers as shown in Figure 4. In the example we examine the effect of the angle $\phi$ on grasp stability. Thus we analyze grasp stability of various objects with various $\phi$ angles. The object and fingers are made of Aluminum $6063-T 5$ with the material properties $\nu=0.33, G=25.86$ [Gpa], and density $\rho=2.7[\mathrm{~g} / \mathrm{ccc}]$. The object's equilibrium configuration is the origin $q_{0}=(0,0,0)^{T} . K_{p}$ and $K_{d}$ are computed according to lemma IV.1, while assuming the following damping coefficients $\frac{\partial \varphi_{i}^{t}}{\partial \dot{\delta}_{i}^{t}}=-1[N \cdot s / m]$ and $\frac{\partial \varphi_{i}^{n}}{\partial \dot{\delta}_{i}^{n}}=$ $-0.01[N \cdot s / m]$. We chose preloading force of $333.615[N]$ (which corresponds to $\delta_{i}^{n}=1 \mu \mathrm{m}$ ) acting along the line connecting the contact points. While applying the stability condition of theorem 1 we identify that the grasp is stable for $\phi<12.68^{\circ}$.

It can be seen from proposition III. 1 that the asymmetry of $K_{i}$ is dominated by $c_{i}=\delta_{i}^{t} / \delta_{i}^{n}$. Enlarging $\phi$ increases $c_{i}$ in order to maintain equilibrium, which in turn, enlarges the asymmetry of $K_{p}$. Therefore, theorem I which bounds the amount of asymmetry of $K_{p}$ practically bounds the angle between the contact force and the normal. For comparison, if we analyze this grasp with the classical convention of Coulomb friction model, taking $\mu=0.3$ (Aluminum on Aluminum), we would get $\phi<16.7^{\circ}$. Our model is conservative as stated in section IV. However, it predicts the same qualitative behavior as the classical Coulomb friction model.

\section{CONCLUSION}

The Hertz-Walton contact model allows concise analytic representation of the contact forces as a function of the contact point displacements. Based on the contact model, we derived contact stiffness matrices, which are asymmetric matrices. As a result the grasp stiffness matrix of the entire grasp is asymmetric. We obtained a concise condition for the global asymptotic stability of the grasp linearized dynamics, and therefore a local asymptotic stability for the nonlinear system. The contact model and the grasp stability analysis seem to extend to $3 D$ grasps under a hard-finger model (i.e. no frictional torque about the contact normal). Finally, we are in process of constructing an experimental grasp arrangement for testing our theoretical predictions [15].

\section{REFERENCES}

[1] W. S. Howard and V. Kumar, "On the stability of grasped objects." IEEE Trans. on Robotics and Automation, vol. 12. no. 6. pp. 904-917, 1996.

[2] Q. Lin, J. W. Burdick. and E. Rimon, "Computation and analysis of natural compliance in grasping and fixturing arrangements," IEEE Trans. on Robotics and Automation (to appear), http://robotics.caltech.edu/papers.

[3] N. Xydas and I. Kao, "Modeling of contact mechanics and friction-limitsurfaces for soft fingers in robotics, with experimental results," The $\mathrm{mt}$. J. of Robotics Research. vol. 18, no. 8. pp. 941-950, 1999.

[4] A. Bicchi, "Hands for dexterous manipulation and robust grasping: A difficult road toward simplicity," IEEE Trans. on Robotics and Automation, vol. 16. no. 6, pp. 652-662. 2000.

[5] P. R. Kraus, V. J. Kumar, and P. Dupont, "Analysis of frictional contact models for dynamic symulation." in IEEE Int. Conf. on Robotics and Automation, Leuven. Belgium, May 1997. pp. 976-981.

[6] Y. Funahashi, T. Yamada, M. Tate. and Y. Suzuki, "Grasp stability analysis considering the curvatures at contact points," in IEEE Int. Conf. on Robotics and Automation, 1996, pp. 3040-3046.

[7] R. D. Mindlin, "Compliance of elastic bodies in contact," Trans. of the $A S M E$, vol. 71. pp. A-259, 1949.

[8] R. D. Mindlin and H. Deresiewicz, "Elastic spheres in contact under varying oblique forces," ASME J. of Applied Mechanics, vol. 20. pp. 327-344. 1953.

[9] K. L. Johnson, Contact Mechanics. Cambridge University Press, 1985.

[10] K. Walton, "The effective elastic moduli of a random packing of spheres," J. of Mech. Physs. and Solids, vol. 35, no. 2, pp. 213-226. 1987.

[11] D. Elata and J. G. Berryman, "Contact force-displacement laws and the mechanical behavior of random packs of identical spheres," Mechanics of Materials, vol. 24, pp. 229-240, 1996.

[12] H. Hertz, "On the contact of elastic solids," in Miscellaneous Papers by H. Hertz (I882). London: Macmillan, 1896.

[13] N. Xydas and 1. Kao, "Study of soft-finger contact mechanics using finite elements analysis and experiments," Manufacturing Automation Laboratory, Dept. of ME, SUNY at Stony Brook, NY," Tech. Report, 2000.

[14] A. Shapiro, E. Rimon, and J. W. Burdick, "On the mechanics of natural compliance in frictional contacts and its effect on grasp stiffness and stability," http://www.technion.ac.il/ robots," Tech. Report, July 2003.

[15] J. W. Burdick, Y. Liang, and E. Rimon, "Experiments in fixturing mechanics," in IEEE Int. Conf. on Robotics and Automation, 2003, pp. $2579-2585$. 